\title{
Original Article \\ Rejection of Trace Level Perfluorohexanoic Acid (PFHxA) in Pure Water by Loose Nanofiltration Membrane
}

\author{
Chenghui Zenga, Shuhei Tanakab, Yuji Suzuki ${ }^{b}$, Satoru Yukioka ${ }^{a}$, Shigeo Fujiib \\ ${ }^{a}$ Graduate School of Engineering, Kyoto University, Kyoto, Japan \\ ${ }^{b}$ Graduate School of Global Environmental Studies, Kyoto University, Kyoto, Japan
}

\begin{abstract}
Recently, perfluorohexanoic acid (PFHxA), an emerging contaminant, has been detected at a high level in the water environment. Its possible presence in drinking water treatment process thus suggests that removal technique should be developed. In this study, one reverse osmosis (RO) membrane, two nanofiltration (NF) membranes and two ultrafiltration (UF) membranes were tested to reject PFHxA (100 - $300 \mathrm{ng} / \mathrm{L})$ in pure water. The measured molecular weight cut-off (MWCO) of two NF membranes were as large as 10,000 and 27,000 Da, while they were still able to reject $96.3 \%$ and 95.3\% PFHxA in pure water, respectively. This indicates PFHxA rejection rate was not dependent on the MWCO of membrane. Results also show that membrane with more negative zeta-potential tends to have higher rejection rate to PFHxA in pure water, suggesting that electrical repulsion between PFHxA and membrane might play an important role in PFHxA rejection. In conclusion, NF membranes would be a better option for removing PFHxA from drinking water than RO membrane because of their larger pure water permeability and $\mathrm{NaCl}$ transmission.
\end{abstract}

Keywords: perfluorohexanoic acid (PFHxA), rejection, nanofiltration (NF), membrane zeta-potential, rejection mechanism

\section{INTRODUCTION}

Perfluoroalkyl and polyfluoroalkyl substances (PFASs) are a group of anthropogenic compounds. Owing to their unique physicochemical properties, including extremely high thermal and chemical stabilities, they have been widely used in industries such as fluoropolymer, semiconductor, and metal plating, etc. These compounds are of growing concern because they are found to be toxic, persistent and bioaccumulative. Some epidemiological studies suggested that the level of certain kinds of PFASs in the blood serum could be linked to low birth weight, infertility-measured as longer waiting time to pregnancy, low semen quality in young men, etc [1-3].

Once the PFASs enter into the environment, they are poorly eliminated. Their transportation through water cycle, atmosphere and food chain leads to the global distribution of PFASs in the environment [4-6]. As a result, human beings could be exposed to the PFASs through various pathways including food, breast milk, drinking water and house dust, etc [7-10]. Before, most of researches had focused on perfluorooctane sulfonate (PFOS) and perfluorooctanoate acid (PFOA) as they were the most common species detected as well as being the final biodegradation products of other PFASs in the natural environment. To provide enough protection to residence from exposure to PFOS and PFOA, the United States Environmental Protection Agency (USEPA) has established health advisory level at $70 \mathrm{ng} / \mathrm{L}$ for PFOS and PFOA or their combination in drinking water in 2016 [11].

Recently, one kind of six carbon chain PFASs - perfluorohexanoic acid (PFHxA) - has been detected as the predominant PFAS in water environment with a maximum concentration reaching as high as $16,000 \mathrm{ng} / \mathrm{L}$ [12]. This implies that some industries have shifted to use PFHxA (short-chain PFAS) as the alternative of PFOA and PFOS (long-chain PFASs), whose usage become less popular due to the more stringent regulation and legislation [13]. Thus, there is a high possibility that presence of PFHxA may be 
Table 1 Membranes used in this study.

\begin{tabular}{llcccc}
\hline \hline Code & Membrane & Type & $\begin{array}{c}\text { Nominal } \\
\text { MWCO (Da) }\end{array}$ & Material & Manufacturer \\
\hline R1 & NTR-759 HR & RO & - & Polyamide & Nitto Denko, \\
N1 & NTR-7410 & NF & 3,000 & Sulfonated polyethersulfone & Japan \\
N2 & NTR-7450 & NF & 1,000 & Sulfonated polyethersulfone & Nadir, \\
\hline U1 & UP020 & UF & 20,000 & Polyethersulfone & Japan \\
U2 & UH030 & UF & 30,000 & Hydrophilic Polyethersulfone & \\
\hline
\end{tabular}

found in drinking water source.

Conventional drinking water treatment process (coagulation, flocculation, sedimentation and sand filtration, etc.) has proven to be ineffective in removal of PFHxA [14]. Effective methods should be developed to remove the PFHxA. The available technologies found today include membrane technology with its ability to reject various kinds of trace organic contaminants in the water. Reverse osmosis applied in drinking water treatment process has been able to reject the PFASs in water almost completely $[15,16]$. The high rejection rates of various kinds of PFASs by nanofiltration (NF) membranes have been reported as well [17]. However, the rejection of PFHxA by membranes, such as reverse osmosis (RO), NF and ultrafiltration (UF), still has not been fully investigated.

The objective of this study was to examine the rejection efficiencies of trace level PFHxA by different membranes. Five commercially available membranes - one RO, two NF and two UF - were tested to reject the PFHxA in pure water. All the tests were conducted in crossflow filtration mode for more than 72 hours in order to get the stable rejection rate to PFHxA in pure water by membranes. Additionally, the membranes were characterized in terms of molecular weight cut-off (MWCO), zeta-potential, pure water permeability and $\mathrm{NaCl}$ rejection rate. The rejection mechanism of PFHxA by membranes was also discussed.

\section{MATERIALS AND METHODS}

\section{Membranes and PFASs standard}

All the reagents used in this study were of HPLC/MS/MS grade unless otherwise specified. PFHxA: $\mathrm{CF}_{3}\left(\mathrm{CF}_{2}\right)_{4} \mathrm{COOH}$ (98\%), methanol and acetonitrile were purchased from Wako Pure Chemical (Osaka, Japan). Oasis ${ }^{\circledR}$ Wax Plus Extraction Cartridge (Waters Corporation, Massachusetts, USA, hereafter referred as Wax cartridge) was used to extract the PFHxA in pure water samples. For MWCO determination, polyethylene glycol (PEG) and polyethylene oxide (PEO) from Wako Pure Chemical and Sigma Aldrich (St. Louis,
USA) were used. In this study, the feed water ( $\mathrm{pH}$ : 7.0) for membrane system was prepared through adding PFHxA into pure water, which was purified by ion exchanger system (Kurita MD-05, Osaka Japan).

Five membranes were used in this study, including RO, NF, and UF membranes. Their characteristics were shown in Table 1. The membranes of Nitto Denko (Osaka, Japan) and Microdyn-Nadir (Wiesbaden, Germany) were purchased from Membrane Soltech (Shiga, Japan) and Mikuni (Osaka, Japan) respectively. All membranes tested here were in flat sheet form. In order to eliminate the impact of compaction during filtration, each membrane was pretreated by filtering pure water under desired transmembrane pressure (TMP).

\section{Membrane set-up}

The membrane set-up was schematically described in Fig. 1. This set-up was a customized product consisting of FTU-1 pressure adjusting part and C-10T crossflow cells (Membrane Soltech, Shiga, Japan) and their details could be found on the website of manufacturer [18]. Basically, the membrane $\left(60 \mathrm{~cm}^{2}\right)$ was housed in the shell $(\mathrm{C}-10 \mathrm{~T})$, simulating the spiral wound membrane system. The crossflow rate and TMP were set at $1 \mathrm{~L} / \mathrm{min}$ and 0.7 or $0.1 \mathrm{MPa}$, respectively. As the membrane set-up was placed in an air-conditioned room, the temperature of feed water became constant after initial several hours of running. All materials used to manufacture the membrane set-up were not adsorptive to the PFHxA so that no adsorption of PFHxA on the membrane system would happen, which is a critical issue when examining the rejection rate of trace level solutes in pure water by membrane.

As shown in Fig. 1, feed water in feed tank 2 was pumped into the feed tank 1 intermittently to compensate the volume reduction in feed tank 1 due to sampling or permeate discharge. Feed water in feed tank 1 was pumped into the shell by a diaphragm pump. The retentate was recycled back to the feed tank, while permeate was discharged or recycled back to the feed tank, depending on the operational conditions. In experiment with membrane R1, N1 and N2, permeate was 


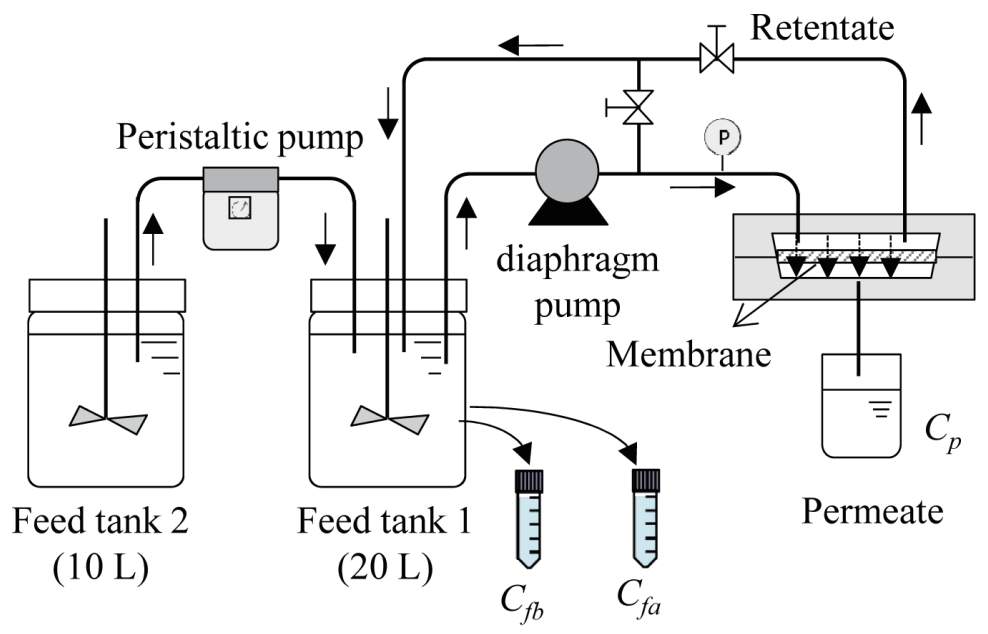

Fig. 1 The membrane experimental set-up used in this study.

discharged in order to repeat the experiment as done before in our lab so that the experimental results could be confirmed. While for experiment with membrane U1and U2, permeate was recycled back to the feed tank. In both of the operational modes, the PFHxA concentration in both experiments was maintained at trace level $(100-300 \mathrm{ng} / \mathrm{L})$, which is the level of PFHxA detected in the surface water. To analyze the PFHxA concentration of permeate, $500 \mathrm{~mL}$ water was sampled at every other 12 hours. However, for feed water, only $50 \mathrm{~mL}$ water was sampled, which was collected before and after the collection of permeate. The average value of them was used to calculate the PFHxA rejection rate as the PFHxA concentration may change slightly before and after the collection of permeate. Meanwhile, the measurement could also be confirmed by twice analysis. The overall rejection rate of PFHxA in water was calculated by following formula:

Rejection rate of PFHxA $(\%)=\left\{1-\frac{C_{p}}{\left(C_{f b}+C_{f a}\right) / 2}\right\} \times 100$

$C_{p}$ : the concentration of PFHxA in permeate (ng/L)

$C_{f b}$ : the concentration of PFHxA in feed sample collected before the collection of permeate sample (ng/L)

$C_{f a}:$ the concentration of PFHxA in feed sample collected after the collection of permeate sample (ng/L)

Based on the results of previous test, the rejection rate of PFHxA would keep fluctuating at initial $12-36$ hours of filtration. In this study, all filtration experiments were conducted for at least 72 hours in order to get the stable rejection rates of each membrane. The rejection rates with variation less than $6 \%$ within 48 hours were regarded as the stable rejection rate.

\section{Analytical method for PFHxA}

PFHxA concentration in water samples were analyzed by solid phase extraction method. Water samples were passed through the WAX cartridge, which was preconditioned by $10 \mathrm{~mL} 0.1 \% \mathrm{NH}_{4} \mathrm{OH}$ in methanol, $10 \mathrm{~mL}$ methanol and $10 \mathrm{~mL}$ MilliQ water in sequence. The flow rate passed through the cartridge was maintained at $10 \mathrm{~mL} / \mathrm{min}$. During this process, the PFHxA was retained inside the cartridge. Then, $4 \mathrm{~mL}$ methanol was used to wash the cartridge and discard in order to remove the matrix in the cartridge. After this, the target compound (PFHxA) was eluted with $2 \mathrm{~mL}$ $0.1 \% \mathrm{NH}_{4} \mathrm{OH}$ methanol solution for 2 times. In total, $4 \mathrm{~mL}$ eluent was collected in a polypropylene tube. The samples were further dried by $\mathrm{N}_{2}$ purge at $60^{\circ} \mathrm{C}$ and reconstituted by $1 \mathrm{~mL} 40 \%$ acetonitrile solution.

Identification and quantification of PFHxA in reconstituted solution were performed by High-performance Liquid Chromatography coupled with tandem mass spectrometer (HPLC/MS/MS, Agilent Technologies, Palo Alto, CA, USA). The separation column used in this study is Zorbax Eclipse Plus C18 (Agilent Technologies, Palo Alto, CA, USA) and the column temperature was set at $40^{\circ} \mathrm{C}$. A mixture of acetonitrile and $5 \mathrm{mmol} / \mathrm{L}$ ammonium acetate solution at a flow rate of $0.25 \mathrm{~mL} / \mathrm{min}$ was used as the mobile phase. The eluent gradient started at $30 \%$ acetonitrile, increased to $50 \%$ at $16.5 \mathrm{~min}$, then to $70 \%$ at $16.6 \mathrm{~min}$, held at $70 \%$ for $3.4 \mathrm{~min}$, went up to $90 \%$ at $21 \mathrm{~min}$, kept at $90 \%$ for $1 \mathrm{~min}$, 
and then ramped down to $30 \%$. The total running time for each sample was $34 \mathrm{~min}$. The average recovery rate of the analytical method was $109 \%$. The instrument detection limit and instrument quantification limit were $0.02 \mathrm{ng} / \mathrm{mL}$ and $0.06 \mathrm{ng} / \mathrm{mL}$, respectively.

\section{MWCO Determination of Membranes}

MWCO was an important parameter to describe the membrane's retention capability. For different membrane manufacturers, the method used to determine the MWCO of membranes may be different. Thus, comparison between nominal MWCO of membranes may not reflect the real pore size differences among membranes used. The main purpose of this experiment was to obtain the MWCO values by the same method so that the pore size of membranes used could be compared. In this study, the experimental set-up described in Fig. 1 with only one feed tank was applied to filter the solution containing $200 \mathrm{mg} / \mathrm{L} \mathrm{PEG}(1-35 \mathrm{kDa})$ or PEO (100 kDa). During filtration, both retentate and permeate were recycled back to the feed tank. The dry membrane coupons obtained from the membrane manufacturer were pre-compacted and washed by filtering with pure water before use. Same crossflow rate and TMP were applied to all membranes. The concentration of PEG/PEO in feed and permeate samples were analyzed by a TOC analyzer (Shimadzu, Kyoto, Japan) in terms of TOC concentration. Following formula was used to calculate the rejection rate of PEG or PEO:

$$
\text { Rejection rate }(\%)=\left(1-\frac{C_{p}}{C_{f}}\right) \times 100
$$

$C_{p}$ : the concentration of PEG/PEO in the permeate sample $(\mathrm{mg} / \mathrm{L})$

$C_{f}$ : the concentration of $\mathrm{PEG} / \mathrm{PEO}$ in the feed sample $(\mathrm{mg} / \mathrm{L})$

\section{Pure water permeability (PWP) and $\mathrm{NaCl}$ rejection measurement}

The experimental set-up for $\mathrm{PWP}$ and $\mathrm{NaCl}$ rejection rate determination was the same with that for MWCO determination. Before the experiment, all membrane coupons were compacted by filtering pure water at $0.7 \mathrm{MPa}$ for at least 1 hour to stabilize the flux. After the compaction, the membrane flux was measured at different TMP ranging from 0.1 to $0.8 \mathrm{MPa}$. The PWP was obtained through the linear regression between membrane flux $\left(J_{w}\right)$ and TMP. Relationship between membrane flux and TMP is expressed as:

$$
J_{w}=\mathrm{PWP} \times \mathrm{TMP}
$$

$J_{w}:$ membrane flux $\left(\mathrm{m}^{3} /\left(\mathrm{m}^{2} \cdot \mathrm{h}\right)\right)$

PWP: pure water permeability $\left(\mathrm{m}^{3} /\left(\mathrm{m}^{2} \cdot \mathrm{h} \cdot \mathrm{MPa}\right)\right)$

TMP: transmembrane pressure $(\mathrm{MPa})$

After the PWP measurement, $\mathrm{NaCl}$ was added into the pure water with a final concentration of $2,000 \mathrm{mg} / \mathrm{L}$ for $\mathrm{NaCl}$ rejection rate determination. The concentration of $\mathrm{NaCl}$ in both feed $\left(C_{s f}\right)$ and permeate $\left(C_{s p}\right)$ was monitored by a conductivity meter (B-173, Horiba, Kyoto, Japan). To stabilize the $\mathrm{NaCl}$ rejection rate, the filtration of $\mathrm{NaCl}$ was conducted for at least 1 hour before measurement. $\mathrm{NaCl}$ rejection rate $(R s)$ and transmission $(T s)$ were expressed by equation (4) and (5):

$$
\begin{aligned}
& R_{s}(\%)=\frac{C_{s f}-C_{s p}}{C_{s f}} \times 100 \\
& T_{s}(\%)=1-R_{s}
\end{aligned}
$$

$R s$ : the $\mathrm{NaCl}$ rejection rate

$C_{s f}$ : concentration of $\mathrm{NaCl}$ in feed $(\mathrm{mg} / \mathrm{L})$

$C_{s p}$ : concentration of $\mathrm{NaCl}$ in permeate $(\mathrm{mg} / \mathrm{L})$

$T s: \mathrm{NaCl}$ transmission

\section{Zeta-potential measurement of membranes}

Zeta-potential is a parameter used to describe the surface charge of membrane. It is well known that zeta-potential could impact the rejection rate of trace organic contaminant by membrane. In this study, the zeta potential of membranes at $\mathrm{pH} 7.0$ were measured with zeta-potential analyzer ELSZ-2000 (Otsuka Electronics Co.,Ltd., Osaka, Japan). Membranes were thoroughly rinsed and soaked in MilliQ water before analysis. The $10 \mathrm{mM} \mathrm{NaCl}$ at $\mathrm{pH} 7.0$ was used as the background electrolyte solution and the zero-charged polystyrene latex was used as the monitor particles. Streaming potential measurement was carried out using solid phase sample cell unit (2000ZS, Otsuka Electronics Co, Ltd., Osaka, Japan) to get the zeta-potential of membranes. The value of membrane zeta-potential was obtained from the computer directly after the automatic analysis of electro-osmosis of polystyrene latex by the coupled software of zeta-potential analyzer. 
a)

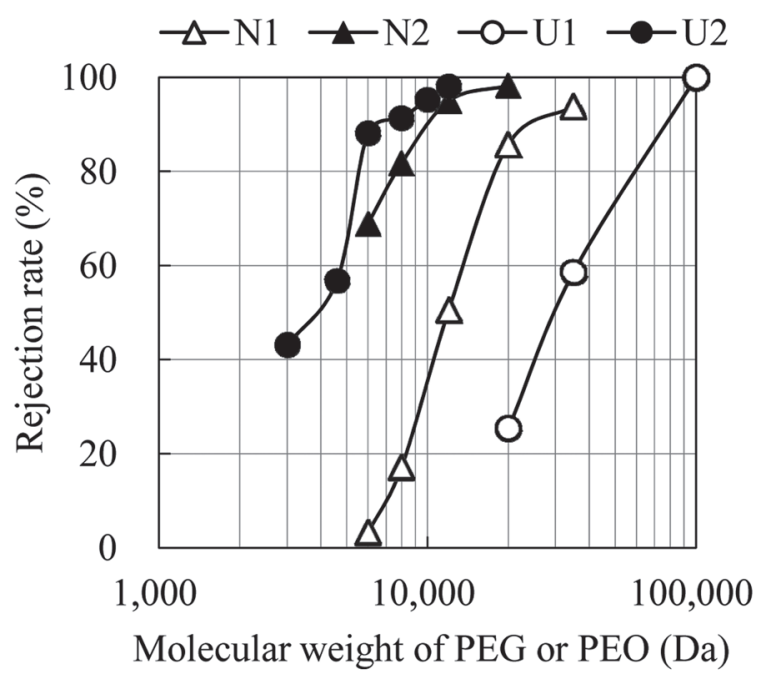

b)

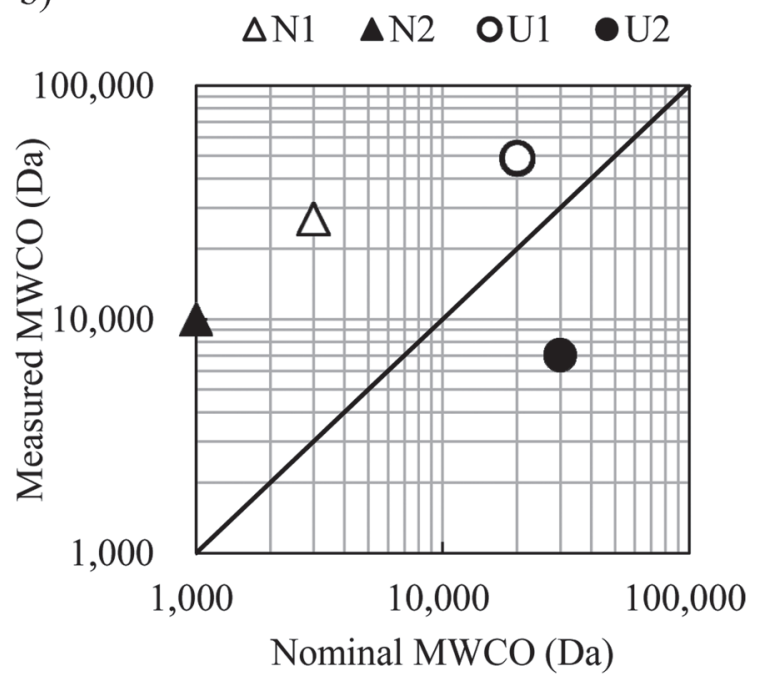

Fig. 2 Results for MWCO determination of membranes (a) Rejection rate of membrane to each kind of PEG or PEO; (b) Comparison between the measured MWCO and nominal MWCO.

\section{RESULTS AND DISCUSSION}

\section{MWCO determination}

MWCO were determined for all membranes except R1 as it was a $\mathrm{RO}$ membrane. The rejection rates of membranes to PEG/PEO were shown in Fig. 2 (a). The MWCO of membrane was defined as the molecular weight of solute which was $90 \%$ rejected by the membrane. Based on this figure, the MWCO of membrane N1, N2, U1 and U2 were estimated as 27,000, 10,000, 49,000 and 7,000 Da, respectively. Fig. 2 (b) showed the comparison between measured MWCO and nominal MWCO. From this figure, it can be found that the measured MWCO of membranes were much larger than their nominal MWCO, except the membrane U2. Currently, there is still no standard method for MWCO determination widely accepted by both membrane manufacturers and researchers. Many factors, such as the types of solutes selected, operational conditions (TMP, crossflow rate and so on.), and filtration modes (crossflow or dead end filtration) used for experiment, could impact the MWCO determination of membrane. Therefore, the discrepancy between the measured MWCO and nominal MWCO may due to the difference of methods used for MWCO determination. It is interesting to note that measured MWCO membrane $\mathrm{N} 1$ and N2 were 27,000, 10,000 Da, respectively. These were much higher than nominal MWCO of NF membranes (200 - 1,000 Da). Thus, they could be, to some extent, regarded as loose NF membranes. $\square \mathrm{R} 1 \Delta \mathrm{N} 1 \Delta \mathrm{N} 2 \quad \mathrm{OU} 1 \bullet \mathrm{U} 2$

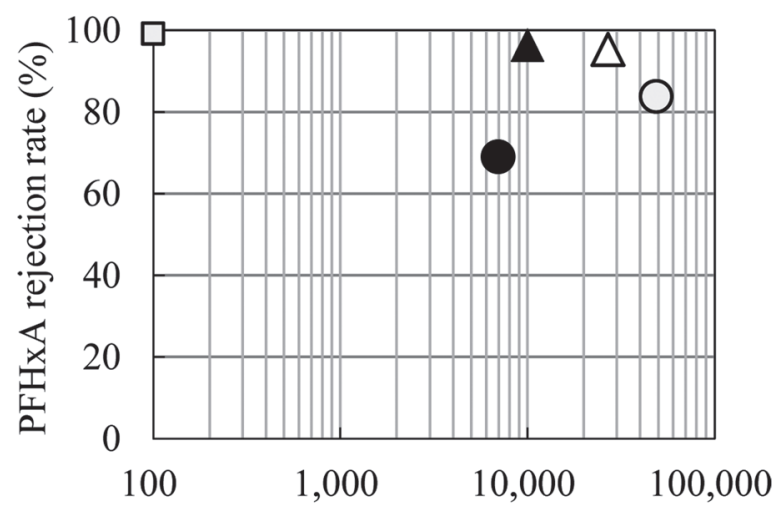

Measured MWCO of each membrane (Da)

Fig. 3 PFHxA stable rejection rate by different membranes.

\section{Stable Rejection Rate of PFHxA by Different Mem- branes}

The stable PFHxA rejection rates of membranes against their respective MWCO values measured in this study were shown in Fig. 3. It can be found that RO membrane (R1) has the highest rejection rate of $99.2 \%$ among all membranes, which is mainly caused by its small membrane pore size (MWCO $<100$ Da, R1 plotted in Fig. 3 was just for comparison with other membranes and this figure did not reflect its real MWCO). This was followed by other two NF membranes - N1 and N2. The measured MWCO of these 


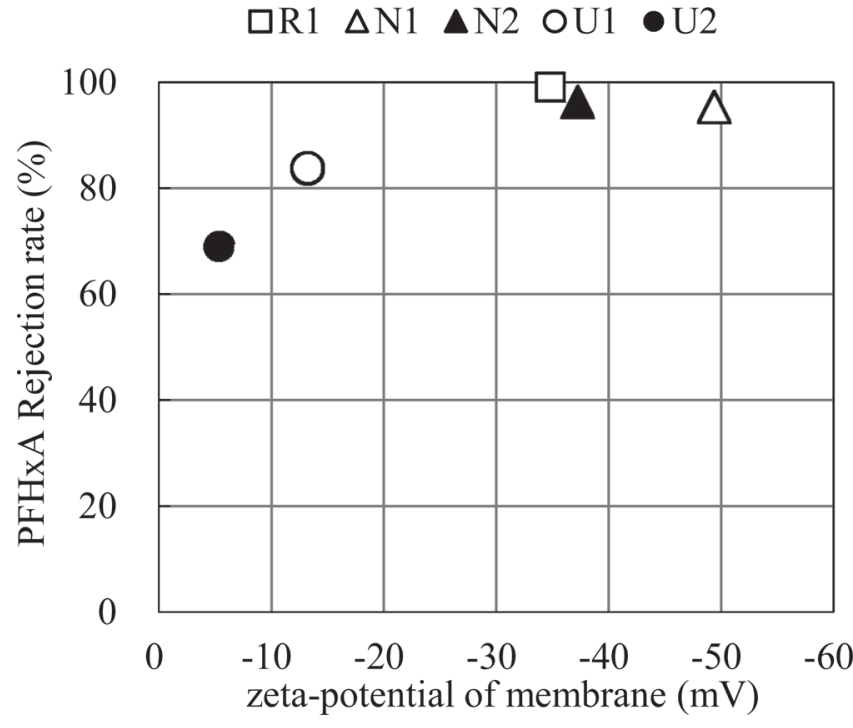

Fig. 4 PFHxA rejection rate V.S. zeta-potential of membranes used.

two membranes were 27,000 and $10,000 \mathrm{Da}$, respectively, while their rejection rates to PFHxA (314 Da) were still as high as $95.3 \%$ and $96.3 \%$, respectively. This suggests that PFHxA rejection rate by membrane does not only depend on the MWCO of membrane. This is further confirmed by the results of membrane U2. Its MWCO was much smaller than that of the two NF membranes, while its rejection rate to PFHxA was only $68.92 \%$. Another interesting finding shows that membrane U1 has a MWCO of 49,000 Da, while its rejection efficiency to PFHxA (314 Da) was still as high as $83.7 \%$. It means that even UF membrane was able to reject most of the PFHxA in pure water.

\section{Zeta-potential and PFHxA Rejection Rate by Mem- branes}

Zeta-potential is an important factor affecting membrane's rejection performance to trace organic contaminants in water. In this study, the measured zeta-potentials for membrane $\mathrm{R} 1, \mathrm{~N} 1, \mathrm{~N} 2$, U1, and $\mathrm{U} 2$ at $\mathrm{pH} 7.0$ were $-34.79,-49.40,-37.23$, -13.21 , and $-5.36 \mathrm{mV}$, respectively. The PFHxA rejection rate versus zeta-potential of membranes used was shown in Fig. 4. From this figure, it can be found that membranes with more negative zeta-potential tend to have higher rejection rate to PFHxA. This suggested that electrical repulsion between the PFHxA and membrane may be another important factor governing the rejection rate of PFHxA by membrane. For NF process, ion rejection was found to be directly related to the membrane charge characteristics [19]. Electrical

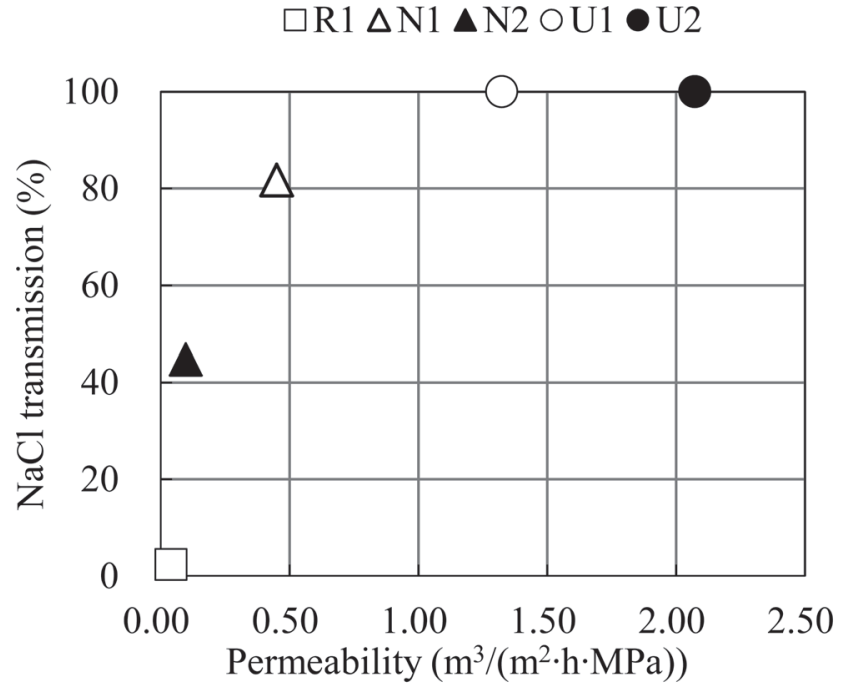

Fig. 5 The $\mathrm{NaCl}$ transmission and the pure water permeability of each membrane.

repulsion between the charged organic solute and membrane can enhance the rejection rate significantly [20]. As shown in Table 1, the selective layers of membrane N1 and N2 were made from sulfonated polyethersulfone. The sulfonate groups on the surface could dissociate and become negatively charged in the water. This leads to the formation of negative zeta-potential of these membranes. As the PFHxA in pure water dissociate into PFHxA ions $\left(\mathrm{CF}_{3}\left(\mathrm{CF}_{2}\right)_{4} \mathrm{COO}^{-}\right)$, charge repulsion between the PFHxA ion and negatively charged membrane takes place. This leads to the high rejection rates of PFHxA by membranes N1 and N2 even though they have very large MWCO. This is in accordance with the findings reported by other researchers. In their study, it was found that once the electrical repulsion effect between PFCs ions and membrane was removed, the rejection rates of PFCs ions reduced dramatically [17]. Thus, membrane zeta-potential might play an important role in the rejection of PFHxA ion in pure water.

For other two UF membranes, their zeta-potentials were less negative than that of RO and NF membranes. The zeta-potential of membrane $\mathrm{U} 2$ was only $-5.36 \mathrm{mV}$, which means its surface was almost neutral. While for another UF membrane U1, its zeta-potential was more negative than that of U2. Thus, it was more negatively charged and it also has higher rejection rate to PFHxA.

\section{The pure water permeability (PWP) and $\mathrm{NaCl}$ rejec- tion rate of membranes}

The PWP and $\mathrm{NaCl}$ transmissions of each membrane were 
shown in Fig. 5. RO membrane $\mathrm{R} 1$ has the lowest $\mathrm{NaCl}$ transmission and PWP among five membranes tested because of its instrinsic high membrane resistance. Unlike RO membrane, NF membranes (N1 and N2) have much higher PWP and $\mathrm{NaCl}$ transmission. This may be due to their large pore size. For UF membranes, the $\mathrm{NaCl}$ transmissions were $100 \%$, which means $\mathrm{NaCl}$ could pass through the UF membranes freely.

Based on above results and discussions, it is found that loose NF membranes (N1 and N2) with very low zetapotential may be a better option for removing PFHxA from drinking water, rather than RO membrane. Reasons are as follows:

(1) N1 and N2 have larger PWP than RO membrane. Thus, their energy consumption would also be lower and cost for drinking water treatment could also be decreased.

(2) The $\mathrm{NaCl}$ transmissions of N1 and N2 were much higher than that of R1. Hence, these two membranes might be less effective in rejecting inorganic ions in water than RO membrane. In other words, minerals in drinking water can pass through these two membranes more easily and this could improve the tap water quality.

Additionally, UF membrane might be another good option for removing PFHxA in drinking water if the water is not so polluted. As shown in Figs. 4 and 5, membrane U1 could reject $83 \%$ PFHxA in pure water. This membrane also has much better flux performance than RO and NF membranes. However, as UF membranes have proven to be much less effective on rejecting other kinds of trace organic contaminant than NF membrane, its application for rejecting PFHxA in drinking water treatment was not recommended here [21,22].

\section{Conclusion}

In summary, the removal efficiencies of trace level PFHxA in pure water by membranes were examined in this study. Main findings of this study are as follows:

1.) Two loose NF membranes were found to have high rejection rates to PFHxA in pure water. These two NF membranes may be better options for removing PFHxA from drinking water than RO membrane due to higher PWP and lower $\mathrm{NaCl}$ rejection rates.

2.) The rejection rates of membranes to PFHxA were not dependent on the MWCO of membranes. Membranes with large MWCO could still have high rejection rate to the PFHxA in pure water.

3.) Membrane with more negative zeta-potential tends to have higher rejection rate to PFHxA. This suggests that electrical repulsion between PFHxA and membrane surface might play an important role in the rejection of PFHxA by membrane.

However, the membranes were only tested with PFHxAcontaining pure water, not in real drinking water treatment process. Thus, the impact of matrix $\left(\mathrm{Ca}^{2+}, \mathrm{Mg}^{2+}\right.$, organic matter, etc.) in drinking water on membrane's performance remains unclear and further investigations are necessary. Last but not the least, the concentrate from the membrane filtration is another problem that needs to be solved.

\section{ACKNOWLEDGEMENT}

This study was supported by JSPS KAKENHI grant number 25289169.

\section{REFERENCES}

[1] Fei C, McLaughlin JK, Tarone RE, Olsen J: Perfluorinated chemicals and fetal growth: a study within the Danish National Birth Cohort. Environ. Health Perspect., 115(11), 1677-1682, 2007. PMID:18008003, doi:10.1289/ehp.10506

[2] Fei C, McLaughlin JK, Lipworth L, Olsen J: Maternal levels of perfluorinated chemicals and subfecundity. Hum. Reprod., 24(5), 1200-1205, 2009. PMID:19176540, doi:10.1093/humrep/den490

[3] Joensen UN, Bossi R, Leffers H, Jensen AA, Skakkebaek NE, Jørgensen N: Do perfluoroalkyl compounds impair human semen quality? Environ. Health Perspect., 117(6), 923-927, 2009. PMID:19590684, doi:10.1289/ehp.0800517

[4] Yamashita N, Kannan K, Taniyasu S, Horii Y, Petrick G, Gamo T: A global survey of perfluorinated acids in oceans. Mar. Pollut. Bull., 51(8-12), 658-668, 2005. PMID:15913661, doi:10.1016/j.marpolbul.2005.04.026

[5] Giesy JP, Kannan K: Peer reviewed: perfluorochemical surfactants in the environment. Environ. Sci. Technol., 36(7), 146A-152A, 2002. PMID:11999053, doi:10.1021/ es022253t

[6] Stock NL, Furdui VI, Muir DC, Mabury SA: Perfluoroalkyl contaminants in the Canadian Arctic: evidence of atmospheric transport and local contamination. Environ. Sci. Technol., 41(10), 3529-3536, 2007. PMID:17547174, doi:10.1021/es062709x

[7] Rahman MF, Peldszus S, Anderson WB: Behaviour and fate of perfluoroalkyl and polyfluoroalkyl substances (PFASs) in drinking water treatment: a review. Water Res., 50, 318-340, 2014. 
[8] Kärrman A, Ericson I, van Bavel B, Darnerud PO, Aune M, Glynn A, Lignell S, Lindström G: Exposure of perfluorinated chemicals through lactation: levels of matched human milk and serum and a temporal trend, 1996-2004, in Sweden. Environ. Health Perspect., 115(2), 226-230, 2007. PMID:17384769, doi:10.1289/ ehp.9491

[9] Tittlemier SA, Pepper K, Seymour C, Moisey J, Bronson R, Cao XL, Dabeka RW: Dietary exposure of Canadians to perfluorinated carboxylates and perfluorooctane sulfonate via consumption of meat, fish, fast foods, and food items prepared in their packaging. J. Agric. Food Chem., 55(8), 3203-3210, 2007. PMID:17381114, doi:10.1021/jf0634045

[10] Post GB, Cohn PD, Cooper KR: Perfluorooctanoic acid (PFOA), an emerging drinking water contaminant: A critical review of recent literature. Environ. Res., 116, 93-117, 2012.

[11] USEPA: Fact Sheet: PFOA and PFOS Drinking Water Health Advisories, https://www.epa.gov/sites/production/files/2016-06/documents/drinkingwaterhealthadvisories_pfop_pfos_updated_5.31.16.pdf [accessed in October, 2016].

[12] Takemine S, Matsumura C, Yamamoto K, Suzuki M, Tsurukawa M, Imaishi H, Nakano T, Kondo A: Discharge of perfluorinated compounds from rivers and their influence on the coastal seas of Hyogo prefecture, Japan. Environ. Pollut., 184, 397-404, 2014.

[13] POPRC: Decision POPRC-1/7: Perfluorooctane Sulfonate; UNEP/POPS/POPRC.1/10; Stockholm Convention on Persistent Organic Pollutants. http://www.pops. int/documents/meetings/poprc/chem_review/PFOS/ PFOS_AnnexD_e.pdf [accessed in October, 2016].

[14] Shivakoti BR, Fujii S, Nozoe M, Tanaka S, Kunacheva $\mathrm{C}$ : Perfluorinated chemicals (PFCs) in water purification plants (WPPs) with advanced treatment processes. Wat. Sci. Technol., 10(1), 87-95, 2010.
[15] Thompson J, Eaglesham G, Reungoat J, Poussade Y, Bartkow M, Lawrence M, Mueller JF: Removal of PFOS, PFOA and other perfluoroalkyl acids at water reclamation plants in South East Queensland Australia. Chemosphere, 82(1), 9-17, 2011. PMID:21051071, doi:10.1016/j.chemosphere.2010.10.040

[16] Radjenović J, Petrović M, Ventura F, Barceló D: Rejection of pharmaceuticals in nanofiltration and reverse osmosis membrane drinking water treatment. Water Res., 42(14), 3601-3610, 2008. PMID:18656225, doi:10.1016/j.watres.2008.05.020

[17] Steinle-Darling E, Reinhard M: Nanofiltration for trace organic contaminant removal: structure, solution, and membrane fouling effects on the rejection of perfluorochemicals. Environ. Sci. Technol., 42(14), 5292-5297, 2008. PMID:18754383, doi:10.1021/es703207s

[18] Membrane Solutions Technology: http://www.membrane-soltech.com/pg178.html [accessed in October, 2016].

[19] Childress AE, Elimelech M: Relating nanofiltration membrane performance to membrane charge (electrokinetic) characteristics. Environ. Sci. Technol., 34(17), 3710-3716, 2000. doi:10.1021/es0008620

[20] Nghiem LD, Schäfer AI, Elimelech M: Role of electrostatic interactions in the retention of pharmaceutically active contaminants by a loose nanofiltration membrane. J. Membr. Sci., 286(1-2), 52-59, 2006. doi:10.1016/j.memsci.2006.09.011

[21] Yoon Y, Westerhoff P, Snyder SA, Wert EC, Yoon J: Removal of endocrine disrupting compounds and pharmaceuticals by nanofiltration and ultrafiltration membranes. Desalination, 202(1-3), 16-23, 2007. doi:10.1016/j.desal.2005.12.033

[22] Mitchell SM, Ullman JL: Removal of Phosphorus, BOD, and Pharmaceuticals by Rapid Rate Sand Filtration and Ultrafiltration Systems. J. Environ. Eng., 142(11), 06016006, 2016. doi:10.1061/(ASCE)EE.19437870.0001137 